\title{
A multilinear interpolation theorem
}

\author{
by
}

MISHA ZAFRAN (Stanford, Calif.)

\begin{abstract}
We prove a general multilinear interpolation theorem for the "espaces de moyenne" of Lions and Peetre. This result is used to obtain a multilinear Marcinkiewicz interpolation theorem.
\end{abstract}

Let $\left(B^{0}, B^{1}\right)$ be an interpolation pair of Banach spaces, and denote by $\left(B^{0}, B^{1}\right)_{s, p}$ (or more simply $\left.B_{s, p}\right), 0<s<1,1 \leqslant p \leqslant \infty$, the real interpolation spaces of Lions and Peetre (see [1], Chapter 3, and [8]). In this paper, we obtain a multilinear interpolation theorem for the spaces $B_{s, p}$ which generalizes a result of the aforementioned authors. As a consequence, we obtain results concerning the real interpolation of operator spaces. In the context of $L_{p}$ spaces, our results, combined with methods closely related to the technique of reiteration (see [1], Chapter 3), yield. a multilinear version of the Marcinkiewicz interpolation theorem. These results are then applied to obtain a Marcinkiewicz-type interpolation theorem for the $H^{p}$ spaces, $0<p<\infty$.

We begin our discussion with some notations and definitions.

1. Let $\left(B^{0}, B^{1}\right)$ be a pair of complex Banach spaces continuously embedded in a topological linear space $\mathscr{r}$. Then $\left(B^{0}, B^{1}\right)$ is called an interpolation pair. If $x \in B^{j}$, we denote its norm by $\|x\|_{j}$ or $\|x\|_{B^{j}}$, $j=0,1$. Under the norm $\|x\|_{B^{0} \cap B^{1}}=\max \left(\|x\|_{0},\|x\|_{1}\right), B^{0} \cap B^{1}$ becomes a Banach space continuously embedded in $\mathscr{V}$. The algebraic sum of $B^{0}$ and $B^{1}$, defined by $\left\{y+z \mid y \in B^{0}, z \in B^{1}\right\}$ and denoted by $B^{0}+B^{1}$, becomes a Banach space continuously embedded in $\mathscr{V}$, when furnished with the norm $\|x\|=\inf \left\{\|y\|_{0}+\|z\|_{1} \mid x=y+z\right.$ and $\left.y \in B^{0}, z \in B^{1}\right\}$.

For any Banach space $X$, we denote by $L_{*}^{p}(X)$ the Banach space of all strongly measurable functions $f$ with domain $(0, \infty)$ and with values in $X$ for which

$$
\|f\|_{L_{*}^{p}(X)}=\left(\int_{0}^{\infty}\|f(t)\|_{X}^{p} \frac{d t}{t}\right)^{1 / p}<\infty \quad \text { if } \quad 1 \leqslant p<\infty
$$

and

$$
\|f\|_{L_{*}^{\infty}(X)}=\underset{t>0}{\operatorname{esssup}}\|f(t)\|_{X}<\infty
$$


where, as usual, we identify functions agreeing almost everywhere. If $X=$ complex numbers, we write $L_{*}^{r}(X)=L_{*}^{r}$.

If $\left(B^{0}, B^{1}\right)$ is an interpolation pair, we define the real interpolation space $\left(B^{0}, B^{1}\right)_{s, p}$ (or $B_{s, p}$ ) to be the collection of all elements $x \in B^{0}+B^{1}$ for which there exists a strongly measurable function $u$ with values in $B^{0} \cap B^{1}$ such that

$$
x=\int_{0}^{\infty} u(t) \frac{d t}{t}
$$

and

$$
t^{-s} u(t) \in L_{*}^{p}\left(B^{0}\right) \quad \text { and } \quad t^{1-s} u(t) \in L_{*}^{p}\left(B^{1}\right) .
$$

It follows by condition (2) that $u \in L_{*}^{1}\left(B^{0}+B^{1}\right)$ so that the integral in (1) is well defined. For $0<s<1$ and $1 \leqslant p \leqslant \infty$, the space $\left(B^{0}, B^{1}\right)_{s, p}$ becomes a Banach space continuously embedded in $\mathscr{V}$ under the norm

$$
\|x\|_{s, p}=\inf \left\{\max \left(\left\|t^{-s} u\right\|_{L_{*}^{p}\left(B^{0}\right)},\left\|t^{1-s} u\right\|_{L_{*}^{p}\left(B^{1}\right)}\right)\right\}
$$
the infimum taken over all $u$ for which $x=\int_{0}^{\infty} u(t) \frac{d t}{t}$ and the condition
(2) above is satisfied.

We recall that there are several other equivalent definitions for the spaces $B_{s, p}$ (see Chapter 3 of [1], [8] and [9]). We use the above definition since it simplifies certain computations in Section 2. The basic properties of these spaces can be found in [1], [8] and [9], and will be used freely throughout.

We now turn to the Lorentz spaces. Let $(\Omega, \Sigma, \mu)$ be a $\sigma$-finite measure space, and let $\mathscr{M}$ denote all complex-valued measurable functions on $\Omega$. Then $\mathscr{M}$ becomes a linear topological space under the topology of convergence in measure on all sets of finite measure in $\Sigma$.

For any $f \in \mathscr{M}$, we let $f^{*}$ denote the non-increasing, right continuous, rearrangement of $f$ (see [6], Section 1). We define the Lorentz space $L_{p, q}(\mu)$ to be the collection of all $f \in \mathscr{M}$ so that $\|f\|_{p, q}^{*}<\infty$, where

$$
\|f\|_{p, q}^{*}=\left\{\begin{array}{lll}
\left(\int_{0}^{\infty}\left(t^{1 / p} f^{*}(t)\right)^{q} \frac{d t}{t}\right)^{1 / q} & \text { if } & 0<p, q<\infty, \\
\sup _{t>0} t^{1 / p} f^{*}(t) & \text { if } & 0<p<\infty, q=\infty .
\end{array}\right.
$$

We define $L_{\infty, q}(\mu)=L_{\infty}(\mu), 0<q \leqslant \infty$.

In general, $\|\cdot\|_{p, q}^{*}$ is not a norm. However, $L_{p, q}(\mu)$ is a metrizable linear topological space (see [6], Section 2). Moreover, if $1<p<\infty$, $1 \leqslant q \leqslant \infty$, then $\|\cdot\|_{p, q}^{*}$ is equivalent to a norm. Specifically, let $f^{* *}(t)$

$$
\begin{aligned}
& =\frac{1}{t} \int_{0}^{t} f^{*}(s) d s . \text { Define } \\
& \|f\|_{p, q}= \begin{cases}\left(\int_{0}^{\infty}\left|t^{1 / p} f^{* *}(t)\right|^{q} \frac{d t}{t}\right)^{1 / q} & \text { if } \quad 1<p<\infty, 1 \leqslant q<\infty, \\
\sup _{t>0} t^{1 / p} f^{* *}(t) & \text { if } \quad 1<p<\infty, q=\infty .\end{cases}
\end{aligned}
$$

Then there exists a constant $C$ (depending only on $p$ and $q$ ) so that $\|f\|_{p, q}^{*}$ $\leqslant\|f\|_{p, q} \leqslant C\|f\|_{p, q}^{*}$, for all $f \in L_{p, q}(\mu)$; the space $L_{p, q}(\mu)$ becomes a Banach space under the norm $\|\cdot\|_{p, q}, 1<p<\infty, 1 \leqslant q \leqslant \infty$ (see [3], [6] or [11], Chapter 5, Section 3). The $L_{p, q}(\mu)$ spaces play an important role in the theory of interpolation of operators. In fact, the weak type condition of Marcinkiewicz is equivalent to a boundedness condition between appropriate Lorentz spaces. We refer the reader to [1] and [11] for this theory. The relation of the $L_{p, q}$ spaces to the abstract interpolation theory of Lions and Peetre may be found in [1], Chapter 3, in case $1<p<\infty$, and in [7], for the full range $0<p<\infty, 0<q \leqslant \infty$. We will assume these results throughout.

2. In this section, we obtain a multilinear interpolation theorem for the real interpolation spaces $B_{s, p}$. This generalizes a theorem of Lions and Peetre (see [8], Chapter 1, 'Theorem 4.1). Our result, combined with interpolation properties of Lorentz spaces will yield a general multilinear Marcinkiewicz interpolation theorem (see Theorem 2.9). We begin with some lemmas.

LEMMA 2.1. Let $\left(B_{k}^{0}, B_{k}^{1}\right),\left(C^{0}, O^{1}\right)$ be interpolation pairs continuously embedded in the.topological linear spaces $\mathscr{V}_{k}$ and $\mathscr{W}$, respectively, $1 \leqslant k \leqslant n$. Let $T$ be a multitinear operator from $\underset{k=1}{\oplus} B_{k}^{0} \cap B_{k}^{1}$ into $C^{0} \cap C^{1}$ such that

$$
\left\|T\left(x_{1}, x_{2}, \ldots, x_{n}\right)\right\|_{C^{j}} \leqslant M_{j} \prod_{k=1}^{n}\left\|x_{k}\right\|_{B_{k}^{j}}
$$

$j=0,1$, for all $\left(x_{1}, x_{n}, \ldots, x_{n}\right) \in \bigoplus_{k=1}^{n} B_{k}^{0} \cap B_{k}^{1}$. Fix $i, 1 \leqslant i \leqslant n$. Then for all $\left(x_{1}, x_{2}, \ldots, x_{n}\right) \in \underset{k=1}{\oplus} B_{k}^{0} \cap B_{k}^{1}$ we have

$$
\left\|T\left(x_{1}, x_{2}, \ldots, x_{n}\right)\right\|_{C^{0}+C^{1}} \leqslant M\left(\prod_{\substack{k=1 \\ k \neq i}}^{n}\left\|x_{k}\right\|_{B_{k}^{0} \cap B_{k}^{1}}\right)\left\|x_{i}\right\|_{B_{i}^{0}+B_{i}^{1}},
$$

where $M=\max \left(M_{0}, M_{1}\right)$.

The proof is a direct consequence of (1), the definitions involved, and by noting that if $x \in B_{i}^{0} \cap B_{i}^{1}$ and $x=a+b$ where $a \in B_{i}^{0}$ and $b \in B_{i}^{1}$, then both $a$ and $b$ are in $B_{i}^{0} \cap B_{i}^{1}$. 
LEMMA 2.2. Assume the notation of the preceding lemma. Let $v_{k} \in$ $L_{*}^{1}\left(B_{k}^{0} \cap B_{k}^{1}\right), 1 \leqslant k \leqslant n-1$, and let $v_{n} \in L_{*}^{1}\left(B_{n}^{0}+B_{n}^{1}\right)$ such that

(a) $v_{n}$ is $B_{n}^{0} \cap B_{n}^{1}$-continuous;

(b) $\int_{0}^{\infty} v_{n}(t) \frac{d t}{t} \in B_{n}^{0} \cap B_{n}^{1}$.

Let $a_{k}=\int_{0}^{\infty} v_{k}(t) \frac{d t}{t}, 1 \leqslant k \leqslant n$. Then

$$
T\left(a_{1}, \ldots, a_{n}\right)=\int_{0}^{\infty} \ldots \int_{0}^{\infty} T\left(v_{1}\left(t_{1}\right), \ldots, v_{n}\left(t_{n}\right)\right) \frac{d t_{1}}{t_{1}} \ldots \frac{d t_{n}}{t_{n}},
$$

the $n$-fold multiple integral converging in $C^{0}+C^{1}$.

Proof. By (a), (b), and the corollary to Theorem 3.7.4 of [5], we see that there exists a sequence $\left\{f_{n, m}\right\}$ of finitely valued, strongly $B_{n}^{0} \cap B_{n}^{1}$ measurable functions such that

$$
\int_{0}^{\infty}\left\|v_{n}(t)-f_{n, m}(t)\right\|_{B_{n}^{0}+B_{n}^{1}} \frac{d t}{t} \rightarrow 0 \text { as } m \rightarrow \infty .
$$

Also, since $v_{k} \in L_{*}^{1}\left(B_{k}^{0} \cap B_{k}^{1}\right), 1 \leqslant k \leqslant n-1$, we see that there exists a sequence $\left\{f_{k, m}\right\}$ of finitely valued, strongly $B_{k}^{0} \cap B_{k}^{1}$-measurable functions such that

$$
\int_{0}^{\infty}\left\|v_{k}(t)-f_{k, m}(t)\right\|_{B_{k}^{0} \cap B_{k}^{1}} \frac{d t}{t} \rightarrow 0 \text { as } m \rightarrow \infty .
$$

The lemma now follows by (1), (2), Lemma 2.1, and since the result evidently holds for functions of the form $f_{k, m}, 1 \leqslant k \leqslant n, 1 \leqslant m<\infty$.

The following lemma can be obtained by transforming Lemma 3.1, Chapter 1 of $[8]$ from $(-\infty, \infty)$ to $(0, \infty)$.

LEMма 2.3. Let $\left(B^{0}, B^{1}\right)$ be an interpolation pair, and let $0<s<1$, $1 \leqslant p \leqslant \infty$. Then if $x \in B_{s, p}$,

$$
\|x\|_{s, p}=\inf \left(\left\|t^{-s} u\right\|_{L_{*}^{p}\left(B^{0}\right)}^{1-s}\left\|t^{1-s} u\right\|_{L_{*}^{p}\left(B^{1}\right)}^{s}\right),
$$

the infimum taken over all strongly measurable $B^{0} \cap B^{1}$-valued functions $u$ satisfying $t^{-s} u \in L_{*}^{p}\left(B^{0}\right), t^{1-s} u \in L_{*}^{p}\left(B^{1}\right)$, and $x=\int_{0}^{\infty} u(t) \frac{d t}{t}$.

We now turn to our general multilinear theorem.

THEOREM 2.4. Let $\left(B_{k}^{0}, B_{k}^{1}\right),\left(C^{0}, O^{1}\right)$ be interpolation pairs, $1 \leqslant k \leqslant n$.
Let $T$ be a multilinear operator from $\bigoplus_{k=1}^{n} B_{k}^{0} \cap B_{k}^{1}$ into $O^{0} \cap O^{1}$ such that

$$
\left\|\boldsymbol{T}\left(x_{1}, \ldots, x_{n}\right)\right\|_{C^{j}} \leqslant M_{j} \prod_{k=1}^{n}\left\|x_{k}\right\|_{B_{k}^{j}},
$$

$j=0,1$, for all $\left(x_{1}, x_{2}, \ldots, x_{n}\right) \in \bigoplus_{k=1}^{n} B_{k}^{0} \cap B_{k}^{1}$. Let $0<s<1,1 \leqslant p_{k} \leqslant \infty$, and suppose $1 / r=\sum_{k=1}^{n} 1 / p_{k}-n+1 \geqslant 0$.

Then

$$
\left\|T\left(x_{1}, \ldots, x_{n}\right)\right\|_{\left(C^{0}, C^{\mathrm{1}}\right)_{s, r}} \leqslant M_{0}^{1-s} M_{1}^{s} \prod_{k=1}^{n}\left\|x_{k}\right\|_{\left(B_{k}^{0}, B_{k}^{1}\right)_{s, p_{k}}},
$$

for all $\left(x_{1}, \ldots, x_{n}\right) \in \bigoplus_{k=1}^{n} B_{k}^{0} \cap B_{k}^{1}$. In particular, if $p_{k}<\infty, 1 \leqslant k \leqslant n$, then $T$ has a unique extension as a bounded multilinear operator from $\bigoplus_{k=1}^{n}\left(B_{k}, B_{k}^{1}\right)_{s, p_{k}}$ into $\left(C^{0}, C^{1}\right)_{s, r}$ of norm $\leqslant M_{0}^{1-s} M_{1}^{s}$.

In the special case $B_{k}^{0} \subseteq B_{k}^{1}, 1 \leqslant k \leqslant n$, this result may be found in [8], Chapter 1, Theorem 4.1.

Proof. For simplicity, we write $\left(C^{0}, C^{1}\right)_{s, r}=C_{s, r}$ and use the somewhat abusive notation $\left(B_{k}^{0}, B_{k}^{1}\right)_{s, p_{k}}=B_{s_{s} p_{k}}$. Since $1 / r=\sum_{k=1}^{n} \frac{1}{p_{k}}-n+1 \geqslant 0$ and $1 \leqslant p_{k} \leqslant \infty$, we see that there exists at most one $k$ such that $p_{k}=\infty$. Hence, without loss of generality, we may assume $1 \leqslant p_{k}<\infty, 1 \leqslant k \leqslant$ $n-1$ and $1 \leqslant p_{n} \leqslant \infty$.

Let $0<\gamma<1$ be fixed, and let $\left(x_{1}, \ldots, x_{n}\right) \in \bigoplus_{k=1}^{n} B_{k}^{0} \cap B_{k}^{1}$. Clearly, we may assume $x_{k} \neq 0$ for all $k$. By [8], Chapter 1, Lemma 2.1, transformed from $(-\infty, \infty)$ to $(0, \infty)$, we see that there exist functions $u_{k}$ with values in $B_{k}^{0} \cap B_{k}^{1}$, and continuous with respect to the norm of $B_{k}^{0} \cap B_{k}^{1}$ such that

$$
\begin{aligned}
& t^{-s} u_{k} \in L_{*}^{p_{k}}\left(B_{k}^{0}\right), \quad t^{1-s} u_{k} \in L_{*}^{p_{k}}\left(B_{k}^{1}\right) \\
& x_{k}=\int_{0}^{\infty} u_{k}(t) \frac{d t}{t}, \\
& \max \left(\left\|t^{-s} u_{k}\right\|_{\left.L_{*}^{p_{k\left(B_{k}^{0}\right.}^{0}}\right)},\left\|t^{1-s} u_{k}\right\|_{L_{*}^{p_{k\left(B_{k}^{1}\right)}}}<(1+\gamma)\left\|x_{k}\right\|_{B_{s, p_{k}}} \cdot\right.
\end{aligned}
$$

Now for $0<\varepsilon<1$, and $1 \leqslant k \leqslant n-1$ define

$$
\dot{u}_{k, \varepsilon}(t)= \begin{cases}u_{k}(t) & \text { if } \quad \varepsilon \leqslant t \leqslant 1 / \varepsilon, \\ 0 & \text { otherwise. }\end{cases}
$$


Define

$$
x_{\varepsilon}^{k}=\int_{0}^{\infty} u_{k, \varepsilon}(t) \frac{d t}{t}
$$

Since $u_{k}$ is $B_{k}^{0} \cap B_{k}^{1}$-continuous, it follows by (2) that the integral defining $x_{e}^{k}$ conterges in the norm of $B_{k}^{0} \cap B_{k}^{1}, 1 \leqslant k \leqslant n-1$.

It is evident that for $0<\varepsilon<1,1 \leqslant k \leqslant n-1$, and $j=0,1$,

$$
\left\|u_{k, \varepsilon}(t)\right\|_{B_{k}^{j}} \leqslant\left\|u_{k}(t)\right\|_{B_{k}^{j}} \quad \text { for all } t \in(0, \infty) .
$$

Thus since $p_{k}<\infty, 1 \leqslant k \leqslant n-1$, we see by the dominated convergence theorem that for $1 \leqslant k \leqslant n-1$,

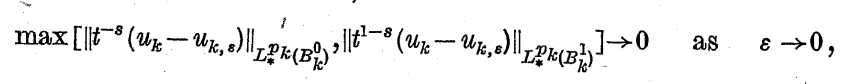

$$
\left\|x_{k}-x_{\varepsilon}^{k}\right\|_{B_{s, p_{k}}} \rightarrow 0 \quad \text { as } \quad \varepsilon \rightarrow 0 \text {. }
$$

Moreover, by (4) we have

(6)

$$
\begin{aligned}
& \left\|t^{-s} u_{k_{k} \varepsilon}\right\|_{L_{*}^{p} k_{\left(B_{k}^{0}\right)}} \leqslant\left\|t^{-s} u_{k}\right\|_{\left.L_{*}^{p} k_{k\left(B_{k}^{0}\right.}^{0}\right)}, \\
& \left\|t^{1-s} u_{k, \varepsilon}\right\|_{L_{*}^{p_{k}}} \leqslant\left\|t^{1-s} u_{k}\right\|_{\left.L_{*}^{p_{k}} k_{\left(B_{k}^{1}\right)}^{1}\right)} .
\end{aligned}
$$

Choose $\varepsilon_{k}$ with $0<\varepsilon_{k}<1,1 \leqslant k \leqslant n-1$. To simplify notation we write

$$
\begin{aligned}
& x_{\varepsilon_{k}}^{k}=a_{k}, 1 \leqslant k \leqslant n-1 \quad \text { and } \quad x_{n}=a_{n}, \\
& u_{k, \varepsilon_{k}}=v_{k}, 1 \leqslant k \leqslant n-1 \quad \text { and } \quad u_{n}=v_{n} .
\end{aligned}
$$

Since, by (2) and (7), the integral defining $a_{k}$ converges in the norm of $B_{k}^{0} \cap B_{k}^{1}, 1 \leqslant k \leqslant n-1$, and since the integral defining $a_{n}$ converges in the norm of $B_{n}^{0}+B_{n}^{1}$ and satisfies the hypotheses of Lemma 2.2, we have

(8) $T\left(a_{1}, a_{2}, \ldots, a_{n}\right)$

$$
=\int_{0}^{\infty} \ldots \int_{0}^{\infty} T\left(v_{1}\left(t_{1}\right), v_{2}\left(t_{2}\right), \ldots, v_{n}\left(t_{n}\right)\right) \frac{d t_{1}}{t_{1}} \frac{d t_{2}}{t_{2}} \ldots \frac{d t_{n}}{t_{n}},
$$

the $n$-fold multiple integral converging in $C^{0}+C^{1}$.

Define a function $w$ as the $(n-1)$-fold multiple integral

(9)

$$
v(t)=\int_{0}^{\infty} \ldots \int_{0}^{\infty} T\left(v_{1}\left(\frac{t}{t_{1}}\right), v_{2}\left(\frac{t_{1}}{t_{2}}\right), \ldots, v_{n-1}\left(\frac{t_{n-2}}{t_{n-1}}\right), v_{n}\left(t_{n-1}\right)\right) \frac{d t_{1}}{t_{1}} \ldots \frac{d t_{n-1}}{t_{n-1}} .
$$

Then by (9), the assumptions concerning the multilinear character and boundedness of $T$, and Young's inequality on convolutions, we obtain

$$
\begin{gathered}
\left\|t^{-s} w\right\|_{L_{*}^{r}\left(C^{0}\right)} \leqslant M_{0}\left\|t^{-s} \int_{0}^{\infty} \ldots \int_{0}^{\infty}\right\| v_{1}\left(\frac{t}{t_{1}}\right) \|_{B_{1}^{0}} \ldots \\
\ldots\left\|v_{n-1}\left(\frac{t_{n-2}}{t_{n-1}}\right)\right\|_{B_{n-1}^{0}}\left\|v_{n}\left(t_{n-1}\right)\right\|_{B_{n}^{0}} \frac{d t_{1}}{t_{1}} \ldots, \frac{d t_{n-1}}{t_{n-1}} \|_{L_{*}^{r}} \\
\leqslant M_{0} \prod_{k=1}^{n}\left\|t^{-s} v_{k}\right\|_{L_{*}^{p_{k}\left(B_{k}^{0}\right)}}<\infty .
\end{gathered}
$$

Similarly,

$$
\left\|t^{1-s} w\right\|_{L_{*}^{r}\left(C^{1}\right)} \leqslant M_{1} \prod_{k=1}^{n}\left\|t^{1-s} v_{k}\right\|_{L_{*}^{p} k_{\left(B_{k}^{1}\right)}}<\infty
$$

Also, it follows by (9), Lemma 2.2, and the translation invariance of the Haar measure $\frac{d t}{t}$ on $(0, \infty)$ that

$$
T\left(a_{1}, a_{2}, \ldots, a_{n}\right)=\int_{0}^{\infty} w(t) \frac{d t}{t} .
$$

Hence $T\left(a_{1}, a_{2}, \ldots, a_{n}\right) \in C_{s, r}$ by (10), (11), and (12). Moreover, by Lemma 2.3, (10), and (11) we have

(13) $\left\|T\left(a_{1}, a_{2}, \ldots, a_{n}\right)\right\|_{s_{s, r}} \leqslant\left(\left\|t^{-s} w\right\|_{L_{*}^{r}\left(C^{0}\right)}\right)^{1-s}\left(\left\|t^{1-s} w\right\|_{L_{*}^{*}\left(C^{1}\right)}\right)^{s}$

$$
\begin{aligned}
& \leqslant\left(M_{0} \prod_{k=1}^{n}\left\|t^{-s} v_{k}\right\|_{L_{*}^{p_{k}\left(B_{k}^{0}\right)}}\right)^{1-s}\left(M_{1} \prod_{k=1}^{n}\left\|t^{1-s} v_{k}\right\|_{L_{*}^{p_{k}\left(B_{k}^{1}\right)}}\right)^{s} \\
& =M_{0}^{1-s} M_{1}^{s} \prod_{k=1}^{n}\left(\left\|t^{-s} v_{k}\right\|_{L_{*}^{p} k_{\left(B_{k}^{0}\right.}^{0}}\right)^{1-s}\left(\left\|t^{1-s} v_{k}\right\|_{\left.L_{*}^{p_{k}\left(B_{k}^{1}\right.}\right)^{s}}{ }^{s} .\right.
\end{aligned}
$$

Therefore, by (1), (6) and (7), it follows that

$$
\left\|T\left(a_{1}, \ldots, a_{n}\right)\right\|_{s_{s, r}} \leqslant M_{0}^{1-s} M_{1}^{s}(1+\gamma)^{n} \prod_{k=1}^{n}\left\|x_{k}\right\|_{B_{s, p_{k}}} .
$$

Thus by (7) and the above inequality,

(14) $\quad\left\|T\left(x_{\varepsilon_{1}}^{1}, x_{\varepsilon_{2}}^{2}, \ldots, x_{\varepsilon_{n-1}}^{n-1}, x_{n}\right)\right\|_{c_{s, r}} \leqslant M_{0}^{1-s} M_{1}^{s}(1+\gamma)^{n} \prod_{k=1}^{n}\left\|x_{k}\right\|_{B_{s, p}}$,

the above inequality valid for $0<\varepsilon_{k}<1,1 \leqslant k \leqslant n-1$. 
Now if $1 \leqslant i \leqslant n-1$ and $0<\alpha, \beta<1$, we define

$$
\nu(i, \alpha, \beta)=\max \left[\left\|t^{-s}\left(u_{i, \alpha}-u_{i, \beta}\right)\right\|_{L_{*}^{p_{i}\left(B_{i}^{0}\right)}},\left\|t^{1-s}\left(u_{i, \alpha}-u_{i, \beta}\right)\right\|_{L_{*}^{p}\left(B_{i}^{1}\right)}\right] .
$$

Note that by (5) we have

$$
\nu(i, \alpha, \beta) \rightarrow 0 \quad \text { as } \quad \alpha, \beta \rightarrow 0 .
$$

By an argument similar to that leading to inequality (14) (see, in particular (13), where now the $i$ th term is treated separately from the other terms in the product), and by (15), we see that for $0<\varepsilon_{k}<1$ and $0<\alpha, \beta$ $<1$,

$$
\left\|T\left(x_{s_{1}}^{1}, \ldots, x_{\alpha}^{i}-x_{\beta}^{i}, \ldots, x_{\varepsilon_{n-1}}^{n-1}, x_{n}\right)\right\|_{s_{s, r}} \leqslant N \nu(i, \alpha, \beta) \prod_{\substack{k=1 \\ k \neq i}}^{n}\left\|x_{k}\right\|_{B_{s, p_{k}}}
$$

for $1 \leqslant i \leqslant \dot{n}-1$, where $N$ may be chosen as $2^{n} M_{0}^{1-s} M_{1}^{s}$ (recall $0<\gamma<1$ ).

Choose $R>0$ such that $\left\|x_{k}\right\|_{B_{s, p_{k}}} \leqslant R$ for $1 \leqslant k \leqslant n$. Then by (17),

$$
\left\|T\left(x_{\varepsilon_{1}}^{1}, \ldots, x_{\alpha}^{i}-x_{\beta}^{i}, \ldots, x_{\varepsilon_{n-1}}^{n-1}, x_{n}\right)\right\|_{C_{s, r}} \leqslant K v(i, \alpha, \beta),
$$

for $0<\varepsilon_{k}<1,1 \leqslant k \leqslant n-1, k \neq i$ and $0<\alpha, \beta<1$, where $K=N R^{n-1}$.

We now show how (14), (16), and (18) yield our theorem. First take $i=1$ in (18). By (16) and (18) we see that $\left\{T\left(x_{a}^{1}, x_{\varepsilon_{2}}^{2}, \ldots, x_{\varepsilon_{n-1}}^{n-1}, x_{n}\right)\right\}$ converges in $C_{s, r}$ as $a \rightarrow 0$, and so converges in $C^{0}+C^{1}$ as $a \rightarrow 0$.

But by $(5), x_{a}^{1} \rightarrow x_{1}$ in $B_{s, p_{1}}$, so $x_{a}^{1} \rightarrow x_{1}$ in $B_{1}^{0}+B_{1}^{1}$. By Lemma 2.1 ,

$$
T\left(x_{a}^{1}, x_{\varepsilon_{2}}^{2}, \ldots, x_{\varepsilon_{n-1}}^{n-1}, x_{n}\right) \rightarrow T\left(x_{1}, x_{\varepsilon_{2}}^{2}, \ldots, x_{\varepsilon_{n-1}}^{n-1}, x_{n}\right)
$$

in $C^{0}+C^{1}$ as $a \rightarrow 0$. Therefore,

$$
T\left(x_{\alpha}^{1}, x_{\varepsilon_{2}}^{2}, \ldots, x_{\varepsilon_{n-1}}^{n-1}, x_{n}\right) \rightarrow T\left(x_{1}, x_{\varepsilon_{2}}^{2}, \ldots, x_{\varepsilon_{n-1}}^{n-1}, x_{n}\right)
$$

as $a \rightarrow 0$, the convergence in $C_{s, r}$.

Note that (19) is valid for any fixed, but arbitrarily chosen $\varepsilon_{k}$ with $0<\varepsilon_{k}<1,2 \leqslant k \leqslant n-1$. Hence by (14) and (19) we obtain

$$
\text { (20) } \quad\left\|T\left(x_{1}, x_{\varepsilon_{2}}^{2}, \ldots, x_{\varepsilon_{n-1}}^{n-1}, x_{n}\right)\right\|_{C_{s, r}} \leqslant M_{0}^{1-s} M_{1}^{s}(1+\gamma)^{n} \prod_{k=1}^{n}\left\|x_{k}\right\|_{B_{s, p_{k}}} \text {. }
$$

We now fix $i \geqslant 2$ in (18), fix $0<\varepsilon_{k}<1$ for $2 \leqslant k \leqslant n-1, k \neq i$, and fix $0<\alpha, \beta<1$. By (19),

(21) $\quad T\left(x_{\varepsilon_{1}}^{1}, x_{\varepsilon_{2}}^{2}, \ldots, x_{\alpha}^{i}-x_{\beta}^{i}, \ldots, x_{\varepsilon_{n-1}}^{n-1}, x_{n}\right)$

$$
\begin{aligned}
=T\left(x_{\varepsilon_{1}}^{1}, \ldots, x_{\alpha}^{i}, \ldots, x_{\varepsilon_{n-1}}^{n-1}, x_{n}\right)-T\left(x_{\varepsilon_{1}}^{1}, \ldots, x_{\beta}^{i}, \ldots, x_{\varepsilon_{n-1}}^{n-1}, x_{n}\right) \\
\rightarrow T\left(x_{1}, x_{\varepsilon_{2}}^{2}, \ldots, x_{\alpha}^{i}-x_{\beta}^{i}, \ldots, x_{\varepsilon_{n-1}}^{n-1}, x_{n}\right) \quad \text { as } \quad \varepsilon_{1} \rightarrow 0,
\end{aligned}
$$

the convergence in $C_{s, r}$.
Hence, letting $\varepsilon_{1} \rightarrow 0$ in (18), we see by (21) that

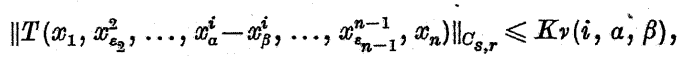

for all $0<\varepsilon_{k}<1,2 \leqslant i \leqslant n-1,2 \leqslant k \leqslant n-1$, and $0<\alpha, \beta<1$.

We now repeat the procedure which led from (14) to (20), and from (18) to (22), now using (22) in place of (18) and (20) in place of (14). The result is

$$
\left\|T\left(x_{1}, x_{2}, x_{\varepsilon_{3}}^{3}, \ldots, x_{\varepsilon_{n-1}}^{n-1}, x_{n}\right)\right\|_{c_{s, r}} \leqslant M_{0}^{1-s} M_{1}^{s}(1+\gamma)^{n} \prod_{k=1}^{n}\left\|x_{k}\right\|_{B_{s, p_{k}}}
$$

(24) $\quad\left\|T\left(x_{1}, x_{2}, x_{\varepsilon_{3}}^{3}, \ldots, x_{\alpha}^{i}-x_{\beta}^{i}, \ldots, x_{\varepsilon_{n-1}}^{n-1}, x_{n}\right)\right\|_{c_{s, r}} \leqslant K v(i, \alpha, \beta)$,

for all $0<\varepsilon_{k}<1,3 \leqslant k \leqslant n-1,3 \leqslant i \leqslant n-1$, and $0<\alpha, \beta<1$. It is clear that after $n-1$ steps we obtain

$$
\left\|T\left(x_{1}, x_{2}, \ldots, x_{n}\right)\right\|_{c_{s, r}} \leqslant M_{0}^{1-s} M_{1}^{s}(1+\gamma)^{n} \prod_{k=1}^{n}\left\|x_{k}\right\|_{B_{s, p_{k}}} .
$$

The theorem follows by (25), the fact that $\gamma>0$ was arbitrary, and by recalling that $B_{s, p_{k}}=\left(B_{k}^{0}, B_{k}^{1}\right)_{s, p_{k}}$.

Comment. The preceding proof could be simplified if we knew the validity of the following result: The elements $x_{\varepsilon}^{k}$ (see equation (3) in the proof of 2.4) satisfy

$$
\left\|x_{\varepsilon}^{k}\right\|_{B_{\delta, p_{k}}}=\inf \left[\left\|t^{-s} v\right\|_{L_{*}^{\left.p_{k\left(B_{k}\right)}\right)}}^{1-s}\left\|t^{1-s} v\right\|_{L_{*}^{p_{k}\left(B_{k}^{1}\right)}}^{s}\right]
$$

the infimum taken over all $B_{k}^{0} \cap B_{k}^{\mathrm{l}}$-valued functions $v$ with compact support in $(0, \infty)$, which are continuous on their support, and which satisfy the additional two properties $x_{\varepsilon}^{k}=\int_{0}^{\infty} v(t) \frac{d t}{t}$ and $t^{j-s} v \in L_{*}^{p_{k}}\left(B_{k}^{j}\right), j=0,1$. (Essentilly this requires the "smooth elements" $x_{\varepsilon}^{k}$ to attain their norm over "smooth functions".) We do not know whether this, in fact, occurs, and anyway, this result does not seem to be of great intrinsic interest.

We remark that Theorem 2.4 plays a crucial role in the construction of multiplier transformations which are of weak type $(p, p)$, but which are not bounded on $L_{p}$. Here $1<p<2$ (see [12]).

Corollary 2.5. Assume the notations of Theorem 2.4. Suppose that $0 \leqslant 1 / q \leqslant \sum_{k=1}^{n} 1 / p_{k}-n+1$. Then there exists a constant $K>0$, depending only on the $p_{k}, q, n$, and $s$, such that for all $\left(x_{1}, \ldots, x_{n}\right) \in \bigoplus_{k=1}^{n} B_{k}^{0} \cap B_{k}^{1}$ we have

$$
\left\|T\left(x_{1}, x_{2}, \ldots, x_{n}\right)\right\|_{\left(C^{0}, C^{1}\right)_{s, q}} \leqslant K M_{0}^{1-s} M_{1}^{s} \prod_{k=1}^{n}\left\|x_{k}\right\|_{\left(B_{k^{3}}^{0} B_{k}^{1}\right)_{s, p_{k}}} .
$$


Proof. Let $1 / r=\sum_{k=1}^{n} 1 / p_{k}-n+1$. The result follows by Theorem 2.4, since for $r \leqslant q,\left(C^{0}, C^{1}\right)_{s, r} \subseteq\left(C^{0}, C^{1}\right)_{s, q}$, the embedding being continuous (see [1], Corollary 3.2.13).

We turn to some applications of Theorem 2.4. As in 2.4, let $\left(B_{k}^{0}, B_{k}^{1}\right)$, $1 \leqslant k \leqslant n,\left(C^{0}, C^{1}\right)$ be interpolation pairs. Let $\mathscr{M}$ denote the space of bounded multilinear mappings $T$ of $\underset{k=1}{n} B_{k}^{0} \cap B_{k}^{1}$ into $C^{0}+C^{1}$ with the norm

$$
\|T\|=\sup \left\|T\left(x_{1}, \ldots, x_{n}\right)\right\|_{C^{0}+C^{1}},
$$

the supremum taken over all $\left(x_{1}, \ldots, x_{n}\right) \in \bigoplus_{k=1}^{n} B_{k}^{0} \cap B_{k}^{1}$ such that $\left\|x_{k}\right\|_{B_{k}^{0} \cap B_{k}^{1}}$ $\leqslant 1,1 \leqslant k \leqslant n$. We further assume $B_{k}^{0} \cap B_{k}^{1}$ is dense in $B_{k}^{j}, j=0,1$, $1 \leqslant k \leqslant n$, and define $\mathscr{M}_{j}$ to be the collection of all bounded multilinear mappings of $\oplus_{k=1}^{n} B_{k}^{j}$ into $C^{j}$ with the norm $\|T\|=\sup \left\|T\left(x_{1}, \ldots, x_{n}\right)\right\|_{C^{j}}$, the supremum taken over all $\left(x_{1}, \ldots, x_{n}\right) \in \oplus_{k=1}^{n} B_{k}^{0} \cap B_{k}^{1}$ such that $\left\|x_{k}\right\|_{B_{k}^{j}}$ $\leqslant 1,1 \leqslant k \leqslant n, j=0,1$.

Then $\mathscr{M}$ is a Banach space and $\mathscr{M}_{0}, \mathscr{M}_{1}$ are Banach spaces continuously embedded in $\mathscr{M}$ (see [2], Section 10.2). We now have the following theorem, which is the analogue for real interpolation spaces of a result of Calderón (see [2], Section 10.2).

THEOREM 2.6. Let $\left(B_{k}^{0}, B_{k}^{1}\right),\left(C^{0}, O^{1}\right)$ be interpolation pairs such that $B_{k}^{0} \cap B_{k}^{1}$ is dense in $B_{k}^{j}, j=0,1,1 \leqslant k \leqslant n$. Let $\mathscr{M}, \mathscr{M}_{0}$ and $\mathscr{M}_{1}$ be as in the preceding paragraph. Let $0<s<1,1 \leqslant q \leqslant \infty, 1 \leqslant l<\infty$, and $1 \leqslant p_{k} \leqslant \infty$. Suppose $0 \leqslant 1 / q \leqslant \sum_{k=1}^{n} 1 / p_{k}+1 / l-n$. Then if $T \in\left(\mathscr{M}_{0}, \mathscr{M}_{1}\right)_{s, l}$, there exists a constant $K>0$, depending only on the $p_{k}, q, l, n$, and $s$, such that

$$
\left\|T\left(x_{1}, \ldots, x_{n}\right)\right\|_{\left(C^{0}, C^{1}\right)_{s, q}} \leqslant K\|T\|_{\left(\mathcal{H}_{0}, M_{1}\right)_{s, l}} \prod_{k=1}^{n}\left\|x_{k}\right\|_{\left(B_{k^{\prime}}^{0}, B_{k}^{1}\right)_{s, p_{k}}},
$$

for all $\left(x_{1}, \ldots, x_{n}\right) \in \underset{k=1}{\oplus} B_{k}^{0} \cap B_{k}^{1}$. In particular, if $p_{k}<\infty$ for all $k$, then $T$ has a unique extension (again denoted by $T$ ) so that (1) remains valid for all $\left(x_{1}, \ldots, x_{n}\right) \in \bigoplus_{k=1}^{n}\left(B_{k}^{0}, B_{k}^{1}\right)_{s, p_{k}}$.

Proof. Let $L\left(x_{1}, x_{2}, \ldots, x_{n}, T\right)=T\left(x_{1}, \ldots, x_{n}\right)$ for all $\left(x_{1}, \ldots, x_{n}, T\right)$ $\underset{k=1}{\in} \oplus_{k}^{n}\left(B_{k}^{0} \cap B_{k}^{1}\right) \oplus\left(\mathscr{M}_{0} \cap \mathscr{M}_{1}\right)$. We now argue as in the proof of 10.2 of [2], using Corollary 2.5.
We now obtain a multilinear analogue of the classical interpolation theorem of Marcinkiewicz. We will adhere to the following notation.

Notation 2.7. Let $\left(\Omega_{k}, \Sigma_{k}, \mu_{k}\right)$ and $(\Omega, \Sigma, \mu)$ denote $\sigma$-finite measure spaces, $1 \leqslant k \leqslant n$. We denote by $\mathscr{S}_{k}$ the integrable simple functions on $\Omega_{k}$, and by $\mathscr{M}$ the measurable functions on $\Omega$. Let $1 \leqslant p_{0}^{k} \neq p_{1}^{k} \leqslant \infty$, $1 \leqslant k \leqslant n, 1 \leqslant q_{0} \neq q_{1} \leqslant \infty$, and $0<s<1$. Define $1 / p_{k}=(1-s) \mid p_{0}^{k}+$ $+s / p_{1}^{k}$ and $1 / q=(1-s) / q_{\theta}+s / q_{1}$. Finally, we recall the definition $L_{\infty, q}$ $=L_{\infty}, 1 \leqslant q \leqslant \infty$.

We require the following preliminary lemma.

LEMMa 2.8. Assume the notations of 2.7 (and Section 1). Let $T$ be a multilinear operator from $\bigoplus_{k=1}^{n} \mathscr{S}_{k}$ into $\mathscr{M}$ such that

$$
\left\|\boldsymbol{T}\left(f_{1}, \ldots, f_{n}\right)\right\|_{q_{q_{j}, \infty}(\mu)}^{*} \leqslant M_{j} \prod_{k=1}^{n}\left\|f_{k}\right\|_{L_{j_{j}, 1}^{k_{k}\left(\mu_{k}\right)}}^{*}
$$

for. all $\left(f_{1}, \ldots, f_{n}\right) \in \bigoplus_{k=1}^{n} \mathscr{S}_{k}, j=0,1$. Then

$$
\left\|T\left(f_{1}, \ldots, f_{n}\right)\right\|_{L_{q, \infty}(\mu)} \leqslant K M_{0}^{1-s} M_{1}^{s} \prod_{k=1}^{n}\left\|f_{k}\right\|_{L_{p_{k}, 1}\left(\mu_{k}\right)},
$$

for all $\left(f_{1}, \ldots, f_{n}\right) \in \bigoplus_{k=1}^{n} \mathscr{S}_{k}$, where $K$ is a constant depending only on $s, n, p_{j}^{k}$ and $q_{j}, 1 \leqslant k \leqslant n, j=0,1$.

Proof. We begin by noting that if $1 \leqslant r \leqslant \infty, 1 \leqslant k \leqslant n$, and if $E \in \Sigma_{k}$ has finite, non-zero $\mu_{k}$-measure, then

$$
\begin{gathered}
\left\|\chi_{E}\right\|_{r, 1}^{*}=r \mu_{k}(E)^{1 / r} \quad \text { if } \quad r<\infty, \\
\left\|\chi_{E}\right\|_{\infty, 1}^{*}=1,
\end{gathered}
$$

where $\chi_{E}$ is the characteristic function of the set $E$. (Recall we define $L_{\infty, 1}$ to be $L_{\infty}$.)

Suppose first $p_{j}^{k}<\infty$ for all $k$ and $j$. Let $E_{k}$ have finite non-zero $\mu_{k}$-measure, $1 \leqslant k \leqslant n$. By (1), it follows easily that

$$
T\left(\chi_{E_{1}}, \ldots, \chi_{E_{n}}\right)^{*}(t) \leqslant O_{j} M_{j} t^{-1 / q_{j}} \prod_{k=1}^{n} \mu_{k}\left(E_{k}\right)^{1 / p_{k}^{j}}
$$

where $C_{j}$ is a constant depending only on the $p_{j}^{k}$ and $n$. (Note this holds even if $q_{0}$ or $q_{1}=\infty$, provided we define $t^{-1 / \infty}=1$.)

Therefore, by (2) we obtain

$$
\begin{aligned}
T\left(\chi_{E_{1}}, \ldots, \chi_{E_{n}}\right)^{*}(t) & =\left[T\left(\chi_{E_{1}}, \ldots, \chi_{E_{n}}\right)^{*}(t)\right]^{1-s}\left[T\left(\chi_{E_{1}}, \ldots, \chi_{E_{n}}\right)^{*}(t)\right]^{s} \\
& \leqslant C M_{0}^{1-s} M_{1}^{s} t^{-1 / q} \prod_{k=1}^{n} \mu_{k}\left(E_{k}\right)^{1 / p_{k}}
\end{aligned}
$$


where $C=C_{0}^{1-s} C_{1}^{s}$. It follows by (1) that

$$
\left\|T\left(\chi_{E_{1}}, \ldots, \chi_{E_{n}}\right)\right\|_{q, \infty}^{*} \leqslant K_{0} M_{0}^{1-s} M_{1}^{s} \prod_{k=1}^{n}\left\|\chi_{E_{k}}\right\|_{p_{k}, 1}^{*},
$$

where $K_{0}$ depends only on $p_{j}^{k}$ and $p_{k}, 1 \leqslant k \leqslant n, j=0,1$. It is also clear that a similar argument applies in case $p_{j}^{k}=\infty$ for some $k$ and $j$, for an appropriate constant $K_{0}$.

Since $1<q<\infty$, we see by [3], Theorem 6 , that there exists $K_{1}>0$, depending only on $q$ and $K_{0}$, so that

$$
\left\|T\left(\chi_{E_{1}}, \ldots, \chi_{E_{n}}\right)\right\|_{L_{q, \infty}(\mu)} \leqslant K_{1} M_{0}^{1-s} M_{1}^{s} \prod_{k=1}^{n}\left\|\chi_{E_{k}}\right\|_{L_{p_{k}, 1}\left(\mu_{k}\right)}
$$

for all $E_{k} \in \Sigma_{k}$ with finite, positive, $\mu_{k}$-measure.

We show that (4) yields the general result by an argument which is well known in the case of linear operators (see [3], Theorem 7). Let $f_{1} \in \mathscr{S}_{1}$ with $f_{1} \geqslant 0$ on $\Omega_{1}$. We write $f_{1}=\sum_{m=1}^{N} a_{m} \chi_{d_{m}}$, where $A_{m} \in \Sigma_{1}$ has finite, nonzero $\mu_{1}$-measure, $a_{m}>0,1 \leqslant m \leqslant N$, and $f_{1}^{*}=\sum_{m=1}^{N} a_{m} \chi_{A_{m}}^{*}$. Then $f_{1}^{* *}=$ $\sum_{m=1}^{N} a_{m} \chi_{A_{m}}^{* *}$ and $\left\|f_{1}\right\|_{L_{p_{1}, 1}\left(\mu_{1}\right)}=\sum_{m=1}^{N} a_{m}\left\|\chi_{A_{m}}\right\|_{L_{p_{1}, 1}\left(\mu_{1}\right)}$. Then by (4),

$$
\begin{gathered}
\left\|T\left(f_{1}, \chi_{E_{2}}, \ldots, \chi_{E_{n}}\right)\right\|_{L_{q, \infty}(\mu)} \leqslant \sum_{m=1}^{N} a_{m}\left\|T\left(\chi_{A_{m}}, \chi_{E_{2}}, \ldots, \chi_{E_{n}}\right)\right\|_{L_{q, \infty}^{*}(\mu)} \\
\leqslant K_{1} M_{0}^{1-s} M_{1}^{s}\left(\prod_{k=2}^{n}\left\|\chi_{E_{k}}\right\|_{\left.L_{p_{k, 1}\left(\mu_{k}\right)}\right)}\right)\left(\sum_{m=1}^{N} a_{m}\left\|\chi_{A_{m}}\right\|_{L_{p_{1}, 1}\left(\mu_{1}\right)}\right) \\
=K_{1} M_{0}^{1-s} M_{1}^{s}\left\|f_{1}\right\|_{L_{p_{1}, 1}\left(\mu_{1}\right)} \prod_{k=2}^{n}\left\|\chi_{E_{k}}\right\|_{L_{p_{k^{1}}\left(\mu_{k}\right)}}
\end{gathered}
$$

where $E_{k} \in \Sigma_{k}$ has finite, non-zero $\mu_{k}$-measure.

Now let $f_{2} \in \mathscr{S}_{2}$ with $f_{2} \geqslant 0$ on $\Omega_{2}$. By the argument leading to (5), now using (5) in place of (4), we obtain

$$
\begin{aligned}
& \left\|T\left(f_{1}, f_{2}, \chi_{E_{3}}, \ldots, \chi_{E_{n}}\right)\right\|_{L_{q, \infty}(\mu)} \\
& \quad \leqslant K_{1} M_{0}^{1-s} M_{1}^{s}\left\|f_{1}\right\|_{L_{p_{1}, 1}\left(\mu_{1}\right)}\left\|f_{2}\right\|_{L_{p_{2}, 1}\left(\mu_{2}\right)} \prod_{k=3}^{n}\left\|\chi_{E_{k}}\right\|_{L_{p_{k}, 1}\left(\mu_{k}\right)},
\end{aligned}
$$

whenever $E_{k} \in \Sigma_{k}$, with $0<\mu_{k}\left(E_{k}\right)<\infty, 3 \leqslant k \leqslant n$. After $n$ steps we
obtain

$$
\left\|T\left(f_{1}, \ldots, f_{n}\right)\right\|_{L_{q, \infty}(\mu)} \leqslant K_{1} M_{0}^{1-s} M_{1}^{s} \prod_{k=1}^{n}\left\|f_{k}\right\|_{L_{p_{k}, 1}\left(\mu_{k}\right)},
$$

for all $f_{k} \in \mathscr{S}_{k}$ with $f_{k} \geqslant 0,1 \leqslant k \leqslant n$.
The lemma follows easily by (7).

Theonem 2.9. Assume the notations of 2.7 (and Section 1). Moreover, suppose $0 \leqslant 1 / q \leqslant \sum_{k=1}^{n} 1 / p_{k}-n+1$. Let $T$ be a multilinear operator from $\bigoplus_{k=1}^{n} \mathscr{S}_{k}$ into $\mathscr{M}$ such that

$$
\left\|T\left(f_{1}, \ldots, f_{n}\right)\right\|_{q_{q_{j}, \infty}(\mu)}^{*} \leqslant M_{j} \prod_{k=1}^{n}\left\|f_{k}\right\|_{p_{j}, 1}^{*}{ }^{\left(\mu_{k}\right)},
$$

for all $\left(f_{1}, \ldots, f_{n}\right) \in \bigoplus_{k=1}^{n} \mathscr{S}_{k}$. Then

$$
\left\|T\left(f_{1}, \ldots, f_{n}\right)\right\|_{L_{q^{(\mu)}}} \leqslant K M_{0}^{1-s} M_{1}^{s} \prod_{k=1}^{n}\left\|f_{k}\right\|_{L_{p_{k}}\left(\mu_{k}\right)},
$$

for all $\left(f_{1}, \ldots, f_{n}\right) \in \bigoplus_{k=1}^{n} \mathscr{S}_{k}$, where $K$ is a constant depending only on the $p_{j}^{k}$, $q_{j}$, n and s. In particular, T has a unique extension to $\bigoplus_{k=1}^{n} L_{p_{k}}\left(\mu_{k}\right)$ satisfying (*).

Proof. We assume $1 \leqslant q_{1}<q_{0} \leqslant \infty$, so that $q_{1}<q<q_{0}$. Choose $q_{0}^{\prime}, q_{1}^{\prime}$ such that $1 \leqslant q_{1}<q_{1}^{\prime}<q<q_{0}^{\prime}<q_{0} \leqslant \infty$. Choose $s_{0}$ and $s_{1}$ satisfying $0<s_{0}<s<s_{1}<1$ and

$$
\frac{1}{q_{j}^{\prime}}=\frac{1-s_{j}}{q_{0}}+\frac{s_{j}}{q_{1}}
$$

$j=0,1$. Let $1<r_{j}^{k}<\infty$ be defined by

$$
\frac{1}{r_{j}^{k}}=\frac{1-s_{j}}{p_{0}^{k}}+\frac{s_{j}}{p_{1}^{k}},
$$

$j=0,1,1 \leqslant k \leqslant n$.

Let $0<\theta<1$ so that $s=(1-\theta) s_{0}+\theta s_{1}$. Then

(3) $\frac{1}{p_{k}}=\frac{1-\theta}{r_{0}^{k}}+\frac{\theta}{r_{1}^{k}}, 1 \leqslant k \leqslant n \quad$ and $\quad \frac{1}{q}=\frac{1-\theta}{q_{0}^{\prime}}+\frac{\theta}{q_{1}^{\prime}}$.

By Lemma 2.8, (1) and (2) we obtain

$$
\left\|T\left(f_{1}, \ldots, f_{n}\right)\right\|_{L_{q_{j}^{\prime}, \infty}(\mu)} \leqslant K_{1} M_{0}^{1-s_{j}} M_{1}^{s_{j}} \prod_{k=1}^{n}\left\|f_{k}\right\|_{L_{r_{j}^{k}, 1}^{k}\left(\mu_{k}\right)},
$$

$j=0,1$, for all $\left(f_{1}, \ldots, f_{n}\right) \in \bigoplus_{k=1}^{n} \mathscr{S}_{k}$, where $K_{1}$ is an appropriate constant. It is easy to see that $T$ has a unique extension so that (4) remains valid for all $\left(f_{1}, \ldots, f_{n}\right) \in \bigoplus_{k=1}^{n} L_{r_{0}^{k}, 1} \cap L_{r_{1}^{k}, 1}$. (Note that this holds even if $r_{0}^{k}$ or $r_{1}^{k}$ equals $\infty$.)

Since $I_{a_{j}^{\prime}, \infty}(\mu)$ and $I_{r_{j}^{k}, 1}\left(\mu_{k}\right)$ are Banach spaces, Theorem 2.4 (and Corollary 2.5) are directly applicable. Thus by (4), the interpolation 
properties of Lorentz spaces (see [1], Chapter 3, Theorem 3.3.10), and by the definition of $\theta$ we obtain

$$
\left\|T\left(f_{1}, \ldots, f_{n}\right)\right\|_{L_{q}(\mu)} \leqslant K M_{0}^{1-s} M_{1}^{s} \prod_{k=1}^{n}\left\|f_{k}\right\|_{L_{p_{k}}\left(\mu_{k}\right)},
$$

for all $\left(f_{1}, \ldots, f_{n}\right) \in \bigoplus_{k=1}^{n} \mathscr{S}_{k}$, where $K$ is as in the statement of the theorem. This completes the proof.

3. In this section, we obtain a Marcinkiewicz-type interpolation theorem for the $H^{p}$ spaces, $0<p<\infty$. Our result is analogous to the Riesz-type theorem for these spaces, as obtained by Calderón and Zygmund (see [13], Chapter 12, Section 3). In the theorem of these aforementioned authors, the point of departure is a multilinear analogue of the Riesz convexity theorem. Similarly, the basis of our result is the multilinear theory of Section 2. Our principal result, Theorem 3.2, was obtained some years ago. In the intervening period, this theorem has been subsumed by a much stronger result of Fefferman, Riviere and Sagher [4]. We include a proof primarily as an application of the results of Section 2 , and we will thus be brief in our presentation.

We begin by recalling the definition of the $H^{p}$ spaces. If $0<p<\infty$, we define $H^{p}$ as the collection of all functions $f$, analytic in the unit disc, for which

$$
\|f\|_{H^{p}}=\sup _{\theta \leqslant r<1}\left(\frac{1}{2 \pi} \int_{0}^{2 \pi}\left|f\left(r e^{i \theta}\right)\right|^{p} d \theta\right)^{1 / p}<\infty .
$$

In case $p \geqslant 1, H^{p}$ is isometric and isomorphic to $\left\{f \in L_{p} \mid \hat{f}(n)=0\right.$ for $n<0\}$. The basic facts concerning these spaces may be found in Chapter 7 of [13].

Notation 3.1. Let $(\Omega, \Sigma, \mu)$ denote a $\sigma$-finite measure space, and let $\mathscr{M}$ denote the measurable functions on $\Omega$. Denote by $\mathscr{P}$ the class of all complex polynomials, restricted to the unit disc. For all real numbers $\alpha>0$, we let $[\alpha]$ denote the greatest integer less than or equal to $\alpha$.

TheOREM 3.2. Assume the notations of 3.1. Let $0<p_{0}<p_{1}<\infty$ and $1 \leqslant q_{0} \neq q_{1} \leqslant \infty$. Let $T$ be a linear operator from $\mathscr{P}$ into $\mathscr{M}$ such that

$$
\|T(f)\|_{q_{j}, \infty}^{*} \leqslant M_{j}\|f\|_{H^{p_{j}}},
$$

for all $f \in \mathscr{P}, j=0,1$. Let $0<s<1$, and let $1 / p=(1-s) / p_{0}+s / p_{1}$ $f 1 / q=(1-s) / q_{0}+s / q_{1}$. Suppose $1 / p$ is not a positive integer and that $1 / q$ $\leqslant 1 / p-[1 / p]$. Then

$$
\|T(f)\|_{L_{q^{(\mu)}}} \leqslant K M_{0}^{1-s} M_{1}^{s}\|f\|_{H^{p}}
$$

or all $f \in \mathscr{P}$, where $K$ is a constant depending only on $p_{j}, q_{j}$, and $s, j=0,1$.
We will require three preliminary lemmas.

LEMMA 3.3. Assume the notations of 2.7. Let $1 \leqslant p_{0}^{k}, p_{1}^{k} \leqslant \infty, 1 \leqslant q_{0}$ $\neq q_{1} \leqslant \infty$, and let $0<\theta<1$. Define $1 / p_{k}=(1-\theta) / p_{0}^{k}+\theta / p_{1}^{k}, 1 \leqslant k \leqslant n$, and $1 / q=(1-\theta) / q_{0}+\theta / q_{1}$. Let $T$ be a multitinear operator from $\oplus_{k=1}^{n} \mathscr{S}_{k}$ into $\mathscr{M}$
such that

$$
\left\|T\left(f_{1}, \ldots, f_{n}\right)\right\|_{q_{j}, \infty}^{*} \leqslant M_{j} \prod_{k=1}^{n}\left\|f_{k}\right\|_{\left.L_{p_{j^{k}}^{k}\left(\mu_{k}\right)}\right)},
$$

$j=0,1$, for all $\left(f_{1}, \ldots, f_{n}\right) \in \bigoplus_{k=1}^{n} \mathscr{S}_{k}$. Then

$$
\left\|T\left(f_{1}, \ldots, f_{n}\right)\right\|_{L_{q}, \infty(\mu)} \leqslant K M_{0}^{1-\theta} M_{1}^{\theta} \prod_{k=1}^{n}\left\|f_{k}\right\|_{L_{p_{k}}\left(\mu_{k}\right)}
$$

for all $\left(f_{1}, \ldots, f_{n}\right) \in \bigoplus_{k=1}^{n} \mathscr{S}_{k}$, where $K$ is a constant depending only on $p_{j}^{h}, q_{j}$ and $\theta, 1 \leqslant k \leqslant n, j=0,1$.

The proof of this result is similar to that of the strong type interpolation theorem as proved by Hunt in [6], pp. 266-269. In fact, the proof of our lemma is easier since we consider multilinear rather than sublinear operators, and since the representation (3.5), p. 266 of [6] becomes elementary under our hypotheses. Hunt's proof will also yield more general versions of Lemma 3.3, but we do not pursue these generalizations.

LEMMA 3.4. Assume the notations of 3.1. Let $0<p_{0}, p_{1}<\infty$ and $1 \leqslant q_{0} \neq q_{1} \leqslant \infty$. Let $T$ be a linear operator from $\mathscr{P}$ into $\mathscr{M}$ such that

$$
\|T(f)\|_{q_{j}, \infty}^{*} \leqslant M_{j}\|f\|_{H^{p_{j}}},
$$

for all $f \in \mathscr{P}, j=0,1$. Let $0<\theta<1$, and let $1 / p=(1-\theta) / p_{0}+\theta / p_{1}$, $1 / q=(1-\theta) / q_{0}+\theta / q_{1}$. Then

$$
\|T(f)\|_{L_{q, \infty}(\mu)} \leqslant K M_{0}^{1-\theta} M_{1}^{\theta}\|f\|_{H^{p}}
$$

for all $f \in \mathscr{P}$, where $K$ is a constant depending only on $p_{j}, q_{j}$, and $\theta, j=0,1$.

Proof. The proof of this result is very similar to that of Theorem 3.9, Chapter 12 of [13]. We now use Lemma 3.3 in place of the multilinear Riesz convexity theorem (see Theorem 3.3, Chapter 12 of [13]).

LENMA 3.5. Assume the notations of 3.1. Let $0<p_{0}<p_{1}<\infty$ and $1 \leqslant q_{0} \neq q_{1} \leqslant \infty$. Let $T$ be a linear operator from $\mathscr{P}$ into $\mathscr{M}$ such that

$$
\|T(f)\|_{q_{j}, \infty}^{*} \leqslant M_{j}\|f\|_{H^{p_{j}}},
$$

for all $f \in \mathscr{P}, j=0,1$. Let $0<\theta<1$, and let $1 / p=(1-\theta) / p_{0}+\theta / p_{1}$, $1 / q=(1-\theta) / q_{0}+\theta / q_{1}$. Suppose $1 / q \leqslant 1 / p-[1 / p]$ and that there exists a positive integer $n$ such that $n-1<1 / p_{1}<1 / p_{0}<n$. Then

$$
\|T(f)\|_{L_{\underline{q}}(\mu)} \leqslant K M_{0}^{1-\theta} M_{1}^{\theta}\|f\|_{H^{p}}
$$

for all $f \in \mathscr{P}$, where $K$ is a constant depending only on $p_{j}, q_{j}$, and $\theta, j=0,1$. 
Proof. Again the proof is similar to that of Theorem 3.9, Chapter 12 of [13]. We first extend $T$ uniquely to $H^{p_{1}}\left(\subseteq H^{p_{0}}\right)$ so that (1) remains valid. Define the multilinear mapping $T^{*}$ by

where

$$
T^{*}\left(g_{1}, \ldots, g_{n}\right)=T\left(F_{1} F_{2} \cdots F_{n}\right)
$$

$$
F_{k}(z)=\frac{1}{2 \pi} \int_{0}^{2 \pi} \frac{e^{i t}+z}{e^{i t}-z} g_{k}(t) d t
$$

for all complex-valued, integrable simple functions $g_{k}$ on $(0,2 \pi)$.

By the inequality of $M$. Riesz, it is easy to see that

$$
\left\|F_{k}\right\|_{H^{r}} \leqslant A_{r}\left\|g_{k}\right\|_{L_{r}}
$$

for $1<r<\infty, 1 \leqslant k \leqslant n$. In particular, $F_{k} \in H^{n p_{1}}, 1 \leqslant k \leqslant n$, and $\prod_{k=1}^{n} F_{k}$ $\in H^{p_{1}}$. Thus the operator $T^{*}$ is well-defined. Moreover, by Hölder's inequality, (1) and (4) we see that

$$
\left\|T^{*}\left(g_{1}, \ldots, g_{n}\right)\right\|_{q_{j}, \infty}^{*} \leqslant C M_{j} \prod_{k=1}^{n}\left\|g_{k}\right\|_{L_{n p_{j}}},
$$

$j=0,1$, for all integrable simple functions $g_{k}$ on $(0,2 \pi), 1 \leqslant k \leqslant n$. Here $C$ is a constant depending only on $p_{j}$ and $n, j=0,1$.

We note also that $1 / q \leqslant \sum_{k=1}^{n} 1 / n p-n+1$ (since this latter term is just $1 / p-[1 / p])$. Thus Theorem 2.9 implies that

$$
\left\|T^{*}\left(g_{1}, \ldots, g_{n}\right)\right\|_{L_{\left.q^{(}\right)}} \leqslant K M_{0}^{1-\theta} M_{1}^{\theta} \prod_{k=1}^{n}\left\|g_{k}\right\|_{L_{n p}}
$$

for all integrable simple funetions $g_{k}$ on $(0,2 \pi)$. Here $K$ depends only on $p_{j}, q_{j}$, and $\theta, j=0,1$.

As in the proof of Theorem 3.9, Chapter 12 of [13], we may extend $T^{*}$ to $\bigoplus_{k=1}^{n} L_{n p_{0}} \cap L_{n p_{1}}$ so that $T^{*}$ is still defined by (2) with the functions $F_{F_{k}}$ and $g_{k}$ related by (3), and so that (5) remains valid. The remainder of the proof is identical to the argument in the aforementioned theorem.

We are finally in a position to prove the principal result of this section.

Proof of Theorem 3.2. Since $1 / p$ is not a positive integer, we may choose a positive integer $n$ so that

Choose $p_{0}^{\prime}$ and $p_{1}^{\prime}$ with

$$
n-1<1 / p<n \text {. }
$$

$$
n-1<\frac{1}{p_{1}^{\prime}}<\frac{1}{p}<\frac{1}{p_{0}^{\prime}}<n
$$

and

$$
\frac{1}{p_{1}}<\frac{1}{p_{1}^{\prime}}<\frac{1}{p_{0}^{\prime}}<\frac{1}{p_{0}} .
$$

We now pick $\theta_{j}$ satisfying

$$
\frac{1}{p_{j}^{\prime}}=\frac{1-\theta_{j}}{p_{0}}+\frac{\theta_{j}}{p_{1}}
$$

$j=0,1$. Note that $0<\theta_{0}<s<\theta_{1}<1$. Define $q_{j}^{\prime}$ by the equations

$$
\frac{1}{q_{j}^{\prime}}=\frac{1-\theta_{j}}{q_{0}}+\frac{\theta_{j}}{q_{1}}
$$

$j=0,1$. By Lemma 3.4, we obtain

$$
\|T(f)\|_{L_{j^{\prime}, \infty}^{(\mu)}} \leqslant K_{1} M_{0}^{1-\theta_{j}} M_{1}^{\theta_{j}}\|f\|_{H_{j}^{p_{j}^{\prime}}}
$$

for all $f \in \mathscr{P}, j=0,1$, where $K_{1}$ depends only on the $p_{j}, q_{j}$, and $\epsilon_{j}$.

Now choose $\theta$ with $s=(1-\theta) \theta_{0}+\theta \theta_{1}$. By computation we see

$$
\frac{1}{p}=\frac{1-\theta}{p_{0}^{\prime}}+\frac{\theta}{p_{1}^{\prime}} \quad \text { and } \quad \frac{1}{q}=\frac{1-\theta}{q_{0}^{\prime}}+\frac{\theta}{q_{1}^{\prime}} \text {. }
$$

Hence by (1), (3), (4), and Lemma 3.5,

$$
\|T(f)\|_{L_{q}(\mu)} \leqslant K M_{0}^{1-s} M_{1}^{s}\|f\|_{H^{p}}
$$

for all $f \in \mathscr{P}, j=0,1$, where $K$ is as in the statement of the theorem. This concludes the proof.

Much of the multilinear theory of Section 2 of this work was taken from a portion of the author's doctoral dissertation, written under the direction of Professor J. D. Stafney at the University of California at Riverside. We wish to thank Professor Stafney for his valuable aid and encouragement.

\section{References}

[1] P. Butzer and H. Berens, Semi-groups of operators and approximation, Springer-Verlag, New York 1967.

[2] A. P. Calderón, Intermediate spaces and interpolation, the complex method, Studia Math. 24 (1964), pp. 113-190.

[3] - Spaces between $L_{1}$ and $L_{\infty}$ and the theorem of Marcinkiewicz, ibid. 26 (1966), pp. 273-299.

[4] C. Fefferman, N. Riviere and Y. Sagher, Interpolation between $H^{p}$ spaces: the real method, Trans. Amer. Math. Soc. 191 (1974), pp. 75-81.

[5] E. Hille and R. Phillips, Functional analysis and semi-groups, Amer. Math. Soc., Providence 1968.

[6] R. A. Hunt, On $L(p, q)$ spaces, Enseignement Math. 12 (1966), pp. 249-276. 
[7] P. Kree, Interpolation d'espaces vectoriels qui ne sont ni normés, ni complets. Applications, Ann. Inst. Fourier, Grenoble 17 (1967), pp. 137-174.

[8] J. L. Lions and J. Peetre, Sur une classe d'espaces d'interpolation, Institut des Hautes Études Scientifiques, 19 (1964), pp. 5-68.

[9] J. Peetre, Sur le nombre de paramètres dans la définition de eertains espaces d'interpolation, Ricerche Mat. 12 (1963), pp. 248-261.

[10] E.M. Stein and G. Weiss, An extension of a theorem of Marcinleiewicz and some of its applications, J. Math. Mech. 8 (1959), pp. 263-284.

[11] -, - Introduction to Fourier analysis on Euclidean spaces, Princeton University Press, 1971.

[12] M. Zafran, Multiplier transformations of weak type, Ann. of Math. 101 (1975) pp. 34-44.

[13] A. Zy gmund, Trigonometric series, 2nd edition, Vol. I and II combined, Cambridge University Press, 1968.

DEPARTMENT OF MATHEMATICS

STANFORD, CALIFORNIA, USA

\section{Harmonic analysis on the group of rigid motions of the Euclidean plane*}

by

RICHARD L. RUBIN (Miami, Florida)

Abstract. Aspects of Fourier analysis on $M(2)$ relevant to the study of $L^{p} \mathrm{mul}$ tiplier operators are developed. Relations between multiplier operators on $\boldsymbol{M}(2)$ and $S O(3)$ or $S U(2)$ are studied. Applications are given to transplantation results for Bessel functions.

Introduction. The idea of considering the real line to be a limit of ircles with increasingly large radii has long been used to relate Fourier analysis on the line, $R$, to Fourier analysis on the torus, $T$. In the study of multiplier operators, this idea leads to the following classical theorem: Let $m$ be a continuous function on $R$. Suppose that for each $\lambda>0$, there exists an operator $M_{\lambda}$ acting continuously on $L^{p}(T)$, given by

$$
M_{\lambda} f(x)=\sum_{n=-\infty}^{\infty} m\left(\frac{n}{\lambda}\right) a_{n} e^{i n x}
$$

where $a_{n}$ is the $n$th Fourier coefficient of $f$. Assume that the operator norms $\left\|M_{\lambda}\right\|$ are uniformly bounded. Then $m$ defines a bounded multiplier operator $M$ on $L^{p}(R)([3]$, p. 264).

We wish to generalize this result by replacing the torus, which may be identified with $S O(2)$, with the non-abelian group $S O(3)$, or with its universal covering group $S U(2)$, which is naturally identifiable with the unit sphere in two-dimensional complex space. By a limiting process analagous to the classical passage from the circle to the line, the group $S O$ (3) ean be shown to tend to a non-compact non-abelian group: the group of rigid motions of the Euclidean plane, denoted by $M(2)$.

In this paper, we shall show how Fourier analysis on $M(2)$ is closely

* The author wishes to thank Professor Guido Weiss and Professor Ronald Coifman for their many helpful discussions concerning this work. 